\title{
THE INHERENT APPEAL OF PHYSICALLY CONTROLLED PERIPHERALS
}

\author{
Daniel Johnson'1 (johnson@dstc.edu.au), John Gardner ${ }^{2}$, Janet Wiles ${ }^{1,3}$, \\ Penelope Sweetser ${ }^{1}$, and Kelly Hollingsworth ${ }^{1}$ \\ ${ }^{\prime}$ School of Information Technology and Electrical Engineering; ${ }^{2}$ School of Management; ${ }^{3}$ School \\ of Psychology, University of Queensland
}

\begin{abstract}
Computer game controllers that require the user to interact using physical movements (other than finger movements) are becoming increasingly popular. The present study sought to explore the factors and context which impact upon preferences for particular physically controlled game peripherals. A comprehensive user study was undertaken and both qualitative and quantitative data were analysed. The results highlight the importance of intuitiveness and realism as factors influencing both the degree of control experienced and attitudes towards physically controlled game peripherals.
\end{abstract}

Key words: physically controlled game peripherals, controller, joystick

\section{Introduction}

Increasingly, computer game peripherals that require physical interaction on the part of the player are being manufactured ("physically controlled peripherals' e.g., dance-mats, fishing rods, guns, skateboards). There are many advantages associated with games that step beyond the paradigm of the user interacting with the game via a small hand-held controller. Many researchers (and indeed, parents) have expressed concern about the value of time spent playing video games. When video games require cardio-vascular or gross-muscular activity for success, users are encouraged to undertake more physical exercise than they otherwise might (and thus they are more likely to experience associated health benefits). Also, physically controlled peripherals can provide the user a more intuitive interaction which tends to appeal to experienced game players and may also increase the appeal of games to people who otherwise would not play them. Furthermore, physically controlled peripherals tend to be very good fun.

Although there is a lack of research specific to physically controlled video game peripherals, research in similar fields provides some indication of potential user reactions to such devices. As Jakob [1] points out, the fundamental issue for computer input is to transfer information from the brain of the user to the comnuter. Progress results from increasing the useful

The original version of this chapter was revised: The copyright line was incorrect. This has been corrected. The Erratum to this chapter is available at DOI: 10.1007/978-0-387-35660-0_65 
bandwidth between the user and the machine by attaining faster, more natural and more convenient means of information transmission. One means by which this goal is currently realised is to make input actions as close as possible to the thoughts that motivated those actions [1], [2]. In video games, this process is often facilitated by physically controlled game peripherals that offer the user a more realistic or intuitive interaction. One goal of the current study is to explore whether feedback from users reflects this process.

In the past, the choice of physically controlled peripherals on the home console market was largely limited to light guns and steering wheels. Light guns are usually used by pulling the trigger to fire and pressing a button (or, less often, a foot pedal) to 'reload'. Steering wheels have generally consisted of a wheel covered with a variety of buttons which the user controls in the style of a normal steering wheel. These devices have often included a gear stick (generally mounted to the side of the steering wheel) and foot pedals which act as the accelerator, clutch and brake.

In recent years a much larger variety of devices have appeared, including motor bike handles, snow/skateboards, fishing rods, dance mats, DJ mixing decks, drum kits, and motion sensitive fighting/boxing controllers. Motorbike handle style controllers, allow the user to steer either by rotating the handles horizontally or by tilting the handles vertically, acceleration can be controlled by twisting the hand grip and breaking by squeezing the brake lever. The Snow/Skateboard devices available consist of a board mounted on two small stands. The user is able to interact with games by standing on the board and leaning, thereby tilting the board for left/right directional control (up/down inputs are provided via foot operated buttons located at the front and rear of the board). Fishing rod controllers consist of a motion sensitive device similar to the bottom half of a real fishing rod (a handle with a reel that can be turned on the side). The device reacts to a casting motion on the part of the user and also to left/right/up/down movements. Dance mats are a collection of flat pressure sensors which can be laid out on the floor. The user interacts with them by standing on different sections of the mat. DJ Mixing Deck controllers are made up of a disc (in the style of a record) which can be rotated and a series of buttons. Drum kit controllers are comprised of a series of pressure sensitive pads arranged on top of a small stand. The user interacts by striking the pads with drum-sticks. Motion sensitive fighting controllers consist of a pressure sensitive mat (similar to a dance mat) used in combination with two upright rods which contain infrared sensors. The user interacts with games by standing on certain points on the mat and/or moving their limbs between the rods in order to break the infrared beams.

Some physically controlled peripherals prove remarkably popular; others are largely rejected by users. The current study seeks to explore when 
and why users prefer physically controlled peripherals to standard control pads. The study represents an important initial attempt to explore the field. Thus, the study is designed to gain broad qualitative data regarding the factors and context which lead to users preferring physically controlled peripherals. Two factors which may impact upon users' attitudes towards control devices are previous experience with games and gender. Specifically, it is theorised that standard controllers may appear somewhat threatening to inexperienced users as they are a unique device with a complex array of buttons whereas physically controlled peripherals tend to present a more transparent or intuitive interface. Irrespective of experience level, it is expected (based on [1] and [2]) that the quality of control experienced by a user will be improved by physically controlled game peripherals which offer the user a more realistic or intuitive interaction.

The current study addressed two hypotheses and a research question. It was expected that prior to playing a game inexperienced users will be more attracted to physically controlled peripherals than standard controllers (H1). It was also expected that the quality of control experienced by a user will be improved by physically controlled game peripherals which offer the user a more realistic or intuitive interaction (H2). The study also examined how (if at all) preferences for standard and physically implemented controllers differ across levels of experience and gender (RQ1).

\section{Method}

Volunteers were recruited for the study via email and word of mouth. Users were given the opportunity to play two Sony Playstation 2 games, SSX Snowboarding (an arcade style snowboarding game) and Time Crisis 2 (TC2; a shooting game), with both a standard controller and an alternate control device. The alternate control devices used were a Thrustmaster Freestyler Board (a snowboard shaped device which the user stands on and shifts their weight to control) for SSX, and a Namco G-Con 2 Gun (a hand held imitation pistol which is aimed at the screen) for TC2.

Users were asked a series of questions in order to determine their level of experience with computer games (how often did they play computer games in an average week, had there been a time in the past when they played computer games more often). Users who currently averaged more than an hour a week playing computer games were considered experienced for the purposes of the study. Seventeen experienced users (11 male and 7 female) and sixteen inexperienced users (6 male and 10 female) were tested.

Users were then shown a short video clip (less than 30 seconds) of each game while an experimenter explained the game's basic concept. Users 
were then asked to indicate, for each game, which controller they would prefer to use (standard or physical) and why they thought that controller would be better. Users then played the first game with both the standard and the physical controllers. The functions available in the game and the way each controller worked were explained to users immediately before they used a particular controller for a particular game. The order of games played and controllers used was counterbalanced across gender and experience level. After users had played the first game with both controllers, they were asked which controller they liked better and why. Users then played the second game with each controller and were again asked which controller they liked better and why.

After playing both games and discussing their experience with the experimenter, users were given a questionnaire with five measures of controller preference for each game. Each measure was rated on a 9-point Likert scale with the standard controller at one end-point and the physical controller at the other end-point. The mid-point of the scale thus represented a lack of preference for one controller over the other. The five measures assessed the users' controller preference in terms of their game performance, level of control, fun, ease of use, and overall preference.

\section{Results and Discussion}

Users' initial controller preferences were examined to investigate $\mathrm{H} 1$. The results largely supported the hypothesis that inexperienced users tend to be attracted to physically controlled peripherals. After viewing footage of TC2, the vast majority of both experienced and inexperienced users indicated that they would prefer to use the gun (of the 17 inexperienced users one inexperienced female indicated a preference for the standard controller). With regard to SSX, most inexperienced users (10 out of 16) indicated an initial preference for the snowboard. Feedback recorded during the study suggested that the intuitive nature of the physical controllers is part of the reason that inexperienced users are initially more attracted to them (comments made included "the board looks more intuitive" and "the board seems more natural"). Although no specific hypotheses were developed regarding the initial preferences of experienced users it is interesting to note that while experienced users shared inexperienced users preference for the gun after watching a clip of TC2 (17 of the 18 experienced users indicated a preference for the gun), they differed from inexperienced users in indicating a preference for the standard controller after watching a clip of SSX (14 of the 18 experienced users indicated a preference for the standard controller). 
After playing each game, users responded to open-ended questions about which controllers they preferred and the reasons for that preference. Comments were categorised into four major themes; issues of ease of control (control), amount of fun (fun), the inherent intuitiveness of the controller (intuitiveness), and the degree of realism engendered by the controller (realism). The theme of 'control' was drawn from comments made about the relative ease or difficulty associated with using the particular controller to interact with the game (e.g., "easier to use", "hard to balance", "moved too much from side to side"). The theme of 'fun' emerged from comments made about the level of enjoyment or pleasure derived from using a particular controller (e.g., "tedious, wasn't fun at all", "more fun"). The theme of 'intuitiveness' was derived from comments made regarding the degree to which the controller was naturally or innately understandable and also the relative amount of practice required to become proficient with the controller (e.g., "more intuitive... easier to adapt to", "just play the game and don't have to worry about how to move"). The theme of 'realism' was drawn from comments made regarding how accurately the controller simulated the equivalent behaviour in real life (e.g., "would push back on you in real life", "not in any way realistic"). Thus, the comments made by users seem likely to allow exploration of $\mathrm{H} 2$ - that the quality of control experienced by a user will be improved by physically controlled game peripherals which offer the user a more realistic or intuitive interaction. In order to explore this hypothesis further, the comments made by users were compared across games and controller types.

When discussing TC2 and the standard controller, users commented most frequently on the lack of ease of control and the lack of intuitiveness. In comparison, the physical controller (gun) was described in terms of ease of control, intuitiveness, fun, and realism. When discussing SSX and the standard controller, users referred most frequently to the ease of control it provided and the fact that it was intuitive. In contrast, the physical controller (board) was described in terms of a lack of ease of control, the absence of an intuitive interface, a lack of realism, but nonetheless as providing good fun. It is interesting to note that (in the case of the snowboard and SSX) fun was reported to exist when using a device that was also considered to be hard to control, lacking an intuitive interface and unrealistic. This suggests the possibility of a novelty value that in some ways counterbalances the less appealing features of the snowboard. It seems plausible that this possibility of a novelty value extends to all physically controlled peripherals as fun was also frequently mentioned when describing the gun, but was rarely mentioned when discussing the standard controller for either game.

The qualitative findings indicate that physically implemented controllers do not always offer a greater quality of control than standard 
controllers, nor are they necessarily more realistic or intuitive. The findings across the two games support the first hypothesis, that the quality of control experienced by a user will be improved by physically controlled game peripherals which offer the user a more realistic or intuitive interaction. When discussing the use of the gun with TC2 (a device described as being easy to control) users made frequent mention of the intuitiveness and realism offered by the device. Contrastingly, when discussing the use of the board with SSX (a device described as being difficult to control) users made frequent mention of the lack of intuitiveness and realism.

It is important to note that while physically controlled peripherals seem to consistently lead to fun on the part of the user they do not necessarily lead to greater ease or quality of control. On the basis of the current findings it seems that in order to improve the quality of control offered to the user it is important that physically controlled peripherals offer more than a similarity to their real life counterparts, specifically, they should provide an intuitive interface and realism. However, where these requirements of intuitiveness and realism are met, there is general support for the idea that in games (as with other software) progress can be made towards the goal of transferring information from the users' brain to the console (or computer) by making the input actions similar to the thoughts which motivated the actions [1], [2].

In order to add further depth to the qualitative analysis undertaken and to allow exploration of the research question regarding how (if at all) preferences for standard and physically implemented controllers differ across levels of experience and gender, quantitative analyses of users' postgame controller preferences were performed. A series of analyses of variance (ANOVA) were conducted on each of the five dependant preference measures. For each ANOVA, there were two between-subjects independent variables: sex and current experience with computer games. Game played (SSX, TC2) was the single within-subjects variable. The analyses thus provided measures of the effects of sex, experience and game played on each preference measure.

For the level of control measure, there was a significant effect for game played $(\mathrm{F}(1,30)=135.03, \mathrm{p}<.001)$. Users reported better control with the physical controller (gun) for TC2 (Mean = 7.03), but reported better control for the standard controller for SSX (Mean = 2.59). For the fun measure, there was a significant effect for game played $(F(1,30)=37.63$, p $<.001$ ). For TC2, users reported more fun for the physical controller (gun) (Mean $=8.09)$. For SSX, users were undecided between the controllers (Mean $=5.35$, which is close to the scale the midpoint of 5). This analysis also showed a significant game-played-by-sex interaction $(F(1,30)=4.66, p$ $=.039$ ). For SSX, females found the physical controller (board) more fun 
$($ Mean $=6.24)$ while males found the standard controller more fun $($ Mean $=$ 4.47). For the performance measure there was a significant effect for game played $(\mathrm{F}(1,30)=160.51, \mathrm{p}<.001)$. Users reported better performance with the physical controller (gun) for TC2 (Mean $=7.26$ ), but reported better performance with the standard controller for SSX (Mean $=2.06)$. This analysis also showed a significant game-played-by-sex interaction $(\mathrm{F}(1,30)=$ $4.28, \mathrm{p}=.047)$. For SSX, females' preferences (Mean $=2.71$ ) were less pronounced than males (Mean $=1.41$ ). This finding is perhaps related to females' lower self-perceptions of performance (on average females had lower ratings of self performance than males on both games), which meant they experienced less of a difference between controller types. Further research would need to assess actual game performance to check this. For the ease of use measure, there was a significant effect for game played $(\mathrm{F}(1,30)=143.68, \mathrm{p}<.001)$. Users reported greater ease of use for the physical controller (gun) for TC2 (Mean $=7.35$ ), but reported greater ease of use for the standard controller for SSX (Mean $=2.47$ ). For the overall controller preference measure, there was a significant effect for game played $(\mathrm{F}(1,30)=108.15, \mathrm{p}<.001)$. Users overall preference was for the physical controller (gun) for TC2 (Mean $=8.00$ ), but they preferred the standard controller for SSX (Mean $=4.15$ ). There was a significant game-played-byexperience interaction $(\mathrm{F}(1,30)=6.16, \mathrm{p}=.019)$. For SSX, inexperienced users were undecided (Mean $=5.31$ ), while experienced users preferred the standard controller (Mean $=3.11$ ). There was also a significant gameplayed-by-sex interaction $(\mathrm{F}(1,30)=7.33, \mathrm{p}=.011)$. For SSX, females were undecided $($ Mean $=5.06$ ), while males preferred the standard controller (Mean $=3.24)$. Overall, the ANOVA results suggest a consistent set of findings for TC2, and more complex patterns for SSX. For TC2, the physical controller (gun) was preferred over the standard controller for game performance, level of control, fun, ease of use, and overall preference. For SSX, the standard controller was generally preferred, except for ratings of fun. Also, ratings made by inexperienced and female users tended to be more equivocal than those made by experienced and male users respectively.

These results add support to the qualitative findings regarding the second hypotheses in that physical controllers are not always preferred, and controller preference depends on the specific type of controller and game involved. When playing TC2 the physically controlled peripheral (the gun) was consistently preferred to the standard controller, but when playing SSX the standard controller was most often preferred to the physically controlled peripheral (the board). As with the qualitative responses from users, when playing SSX the board was preferred over the standard controller in terms of fun. The results also indicated some preference differences based on sex and experience. Males and more experienced users consistently preferred the 
standard controller to the board, while females and less experienced users were more equivocal in their ratings. This finding is possibly related to differing performance goals across experience levels and gender. If males and experienced users have higher performance expectations when playing video games then they are likely to globally favour the device with which they perform better. In contrast, for females and inexperienced users performance in the game may not be as important in terms of preference as the novelty and fun experienced with the device.

\section{Conclusions}

The study represents an important initial exploration of the factors contributing to users' preferences for physically controlled game peripherals. Overall, the results indicated that controller preferences are not simply determined by the nature of the device (standard versus physical) but instead that preferences are moderated by amount of control, intuitiveness and degree of realism experienced, and also by demographic factors of gender and experience level. A number of useful directions for future research can be identified. Obviously, greater generalisation of the results should be sought by testing a greater quantity and variety of games, physically controlled peripherals, and people. Moreover, a deeper understanding of the factors which contribute to the inherent realism or intuitiveness of controllers could be achieved via semi-structured interviews with users.

Acknowledgement: We gratefully acknowledge the support of the Distributed Systems Technology Centre (http://www.dstc.edu.au)

\section{References}

[1] Jakob, R.J.K. (1996). Human-computer interaction: Input devices. ACM Computing Surveys, 28(1), 177-179.

[2] Hutchins, E.A.. Hollan, J.D., and Norman, D.A.(1986). Direct manipulation interfaces. In User Centred System Design: New Perspectives in Human-computer Interaction. D.A. Norman and S.W. Draper (Eds.), Lawrence Erlbaum, Hillsdale, NJ, 87-124. 


\section{VIRTUAL REALITY TECHNOLOGIES FOR ENTERTAINMENT}

